\title{
On the Uses and Applications of the Most Commonly Used Measures of Linkage Disequilibrium from the Comparative Analysis of Their Statistical Properties
}

\author{
Carlos Zapata \\ Departamento de Genética, Universidad de Santiago, Santiago de Compostela, Spain
}

\section{Key Words \\ Linkage disequilibrium • Measures of disequilibrium • $D^{\prime}$ and $r$ coefficients - Sampling distributions - Sampling variance and bias - Range of disequilibrium measures . $2 \times 2$ contingency tables}

\begin{abstract}
Background/Objective: The analysis of linkage disequilibrium is relevant for the exploration of the structure and evolution of genomes and for the gene mapping of quantitative characters and human diseases. The strength of linkage disequilibrium between diallelic loci is commonly measured by the coefficients $D^{\prime}$ and $r$. Recent studies suggest that $r$ is more useful than $D^{\prime}$ as a general measure of the strength of disequilibrium because it provides much more precise (lower sampling variance) and accurate (lower bias) estimates of disequilibrium. We compared for the first time the statistical properties of $D^{\prime}$ and $r$ taking into account their differences in range. Methods: The sampling properties of $D^{\prime}$ and $r$ were evaluated by simulation under a variety of realistic population conditions and varying sample sizes using standardised statistics that allow for comparisons of the precision, accuracy and efficiency of estimates with different ranges. $\boldsymbol{R e}$ sults: Simulations revealed that estimates of $r$ do not tend to
\end{abstract}

be significantly more precise, accurate or efficient than those of $D^{\prime}$ when compared by means of standardised statistics. Conclusion: The supposed advantage of $r$ over $D^{\prime}$ based on direct comparisons of their sampling distributions is more apparent than real. The obtained results are useful to assess the uses and applications of these widely used disequilibrium measures.

Copyright $\odot 2011$ S. Karger AG, Basel

\section{Introduction}

Estimation of non-random associations of alleles at different loci (LD, linkage disequilibrium) is one of the most important tools for exploring the past and recent influence of evolutionary mechanisms and population factors contributing to shaping the multilocus structure of the genome [1]. The past few years have witnessed an extraordinary surge of information about patterns of LD along genomes greatly stimulated by the application of LD to the positional cloning of genetic factors underlying quantitative characters and human diseases. In particular, considerable effort has been devoted to the generation of detailed maps of the strength of LD between tightly linked single nucleotide polymorphisms (SNPs) scattered

\section{KARGER}

Fax +4161306 1234

E-Mail karger@karger.ch

www.karger.com
C 2011 S. Karger AG, Basel

0001-5652/11/0713-0186\$38.00/0

Accessible online at:

www.karger.com/hhe
Carlos Zapata

Departamento de Genética, Centro de Investigaciones Biológicas de la USC (CIBUS) Universidad de Santiago, Avda. Lope Gómez de Marzoa s/n

ES-15782 Santiago de Compostela (Spain)

Tel. +34818 816922 , E-Mail c.zapata@usc.es 
across all human chromosomes to facilitate the mapping of complex disease loci [2].

The strength of LD between diallelic loci can be estimated by a variety of measures or coefficients, either directly from haplotypes or indirectly from genotype data [3-5]. In practice, $D^{\prime}$ (or $\left|D^{\prime}\right|$ ) [6] and $r$ (or $r^{2}$ ) [7] are the most widely used measures of LD. $D^{\prime}$ and $r$ are standardisations of the covariance of allele frequencies $(D)$ by its maximum possible value for the observed allele frequencies and the product of standard deviations of allele frequencies at the two loci, respectively. However, both standardised coefficients often provide very discrepant estimates of the strength of LD from the same haplotype dataset. Thus, analyses of different population genetic models by simulation $[8,9]$, genome-wide patterns of $\mathrm{LD}$ [9-14] and numerical cases [15] have shown that $r$ (or $r^{2}$ ) values are typically lower than the corresponding $D^{\prime}$. It is often assumed that $D^{\prime}$ and $r$ reflect different aspects of LD and have distinct advantages and disadvantages depending on the objective of the study [16-19].

Differences in the range, precision and accuracy are thought to explain why $D^{\prime}$ and $r$ give such discrepant estimates of LD from the same array of haplotypes. Although there is no LD measure that is completely independent of the allele frequencies at the loci [20], the potential range of variation for $D^{\prime}$ is always between -1.0 and +1.0 , whereas the limits of the range of $r$ show great dependence on the allele frequencies at the two loci $[8$, 20]. On the other hand, the reliability of $D^{\prime}$ and $r$ can be evaluated by their levels of precision and accuracy. Precision depends on the degree of dispersion of the estimates over replicate samples taken from the same population, whereas accuracy is dependent on the degree of proximity between the true population value and the estimates. Recent simulation studies have been conducted to assess the degree of precision and accuracy of estimates of $D^{\prime}$ and/or $r$ in small samples by means of sampling variances and biases, respectively [21-23]. Teare et al. [21] concluded that $D^{\prime}$ tends to give estimates with a larger variance and bias than $r$ due to the normalisation of the covariance of allele frequencies by its maximum value. It was particularly emphasised that estimates of $D^{\prime}$ (in absolute values) tend to be strongly biased in an upward direction under the influence of a number of factors, with the bias increasing for small sample sizes, low allele frequencies and weak LD. In contrast, the same study showed that the bias of estimates of $r$ was often not large in magnitude and was much less affected by sample sizes and population conditions. Similar results were obtained from simulated populations with extreme allele frequen- cies and variable levels of $\mathrm{LD}$ throughout the positive range $[22,23]$.

Numerous authors have recently highlighted that $D^{\prime}$ is less useful than $r$ as a general measure of the strength of LD on the basis of their differences in bias [e.g. 14, 17, $18,21-24]$. Specifically, it is considered that $D^{\prime}$ leads to overestimating the importance of LD in populations because the absolute value of its estimates is strongly upward biased under many realistic scenarios [14, 17, 2123]. It is also considered that the use of $D^{\prime}$ affects the validity of comparisons of the extent of $\mathrm{LD}$ across genomic regions within and between samples of varying sizes because its bias is dependent on the level of polymorphism at the loci, the strength of $\mathrm{LD}$ and the sample sizes $[14,17$, $18,24]$. Nevertheless, the evidence on the relative precision and accuracy of $D^{\prime}$ and $r$ is still inconclusive because studies conducted so far have invariably been based on direct comparisons of the magnitudes of their sampling variances and biases. This approach presents a potential problem because it misses the essential feature that both coefficients often vary in different ranges or scales, which could significantly affect the conclusions.

In this work, we carried out a simulation study on the sampling statistical properties of $D^{\prime}$ and $r$ under a variety of realistic population conditions and varying sample sizes. $D^{\prime}$ and $r$ were chosen for comparative purposes instead of alternative versions of both statistics without sign (i.e. $\left|D^{\prime}\right|$ and $r^{2}$ ) because it was previously shown that the magnitude of the asymptotic sampling variance of $D^{\prime}$ undergoes large sign-dependent variations [25]. The relative performance of both measures was evaluated for the first time by applying standardised statistics, allowing comparisons of the precision and accuracy of estimates with different ranges.

\section{Methods}

Notation and Measures of Disequilibrium

Consider two loci, $A$ and $B$, with each locus having two possible alleles, $A_{1} / A_{2}$ and $B_{1} / B_{2}$, respectively. Let $p, 1-p, q$ and $1-q$ be the population frequencies of the alleles $\left(A_{1}, A_{2}, B_{1}\right.$ and $B_{2}$, respectively) and $p_{11}, p_{12}, p_{21}$ and $p_{22}$ represent the population frequencies of the four possible haplotype classes $\left(A_{1} B_{1}, A_{1} B_{2}, A_{2} B_{1}\right.$ and $A_{2} B_{2}$, respectively). Allele and haplotype frequencies are generally unknown population parameters unless the total population has been scored, and they are directly and indirectly estimated from a random sample of haplotypes and genotypes, respectively. Therefore, relative allele and haplotype frequencies estimated from a population sample of size $N$ are denoted by $\hat{p}$, $1-\hat{p}, \hat{q}, 1-\hat{q}$, and $\hat{p}_{11}, \hat{p}_{12}, \hat{p}_{21}, \hat{p}_{22}$, respectively. Two-locus haplotype frequencies can be arranged in a $2 \times 2$ matrix; therefore, $L D$ 
Table 1. Range and values of $D^{\prime}$ and $r$ under different simulated population conditions

\begin{tabular}{|c|c|c|c|c|c|c|c|}
\hline Measure & $p$ & $q$ & minimum & maximum & $-W /+W$ & $-M /+M$ & $-S /+S$ \\
\hline \multirow{3}{*}{$r$} & 0.7 & 0.5 & -0.655 & +0.655 & $-0.131 /+0.131$ & $-0.327 /+0.327$ & $-0.524 /+0.524$ \\
\hline & 0.9 & 0.5 & -0.333 & +0.333 & $-0.067 /+0.067$ & $-0.167 /+0.167$ & $-0.267 /+0.267$ \\
\hline & 0.7 & 0.7 & -0.429 & +1.000 & $-0.086 /+0.200$ & $-0.214 /+0.500$ & $-0.343 /+0.800$ \\
\hline$D^{\prime}$ & all & all & -1.000 & +1.000 & $-0.200 /+0.200$ & $-0.500 /+0.500$ & $-0.800 /+0.800$ \\
\hline
\end{tabular}

will be positive or negative depending on whether frequencies of haplotype configurations in coupling $\left(A_{1} B_{1}\right.$ and $\left.A_{2} B_{2}\right)$ are in excess or in deficit, respectively, with respect to those expected by random association. It follows that the sign or direction of LD will depend on the criterion chosen to designate the allelic composition of coupling haplotype classes. A homogeneous criterion was then followed to designate haplotype classes in order to compare the properties of estimates of the strength of LD depending on their sign across pairs of loci. Coupling haplotypes were designated as those carrying the most and the least frequent allele variants, in accordance with many previous LD studies [26-29].

The strength of LD was measured using the measures or coefficients $D^{\prime}$ and $r[6,7]$. Estimates of $D^{\prime}$ were obtained by

$$
\hat{D}^{\prime}=\hat{D} / \hat{D}_{\text {max }}
$$

where $\hat{D}=\hat{p}_{11}-\hat{p} \hat{q}$ and $\hat{D}_{\text {max }}$ is the maximum possible value of $\hat{D}$ depending on the relative allele frequencies at the loci and its sign. $\hat{D}_{\text {max }}$ is defined as $\min [\hat{p}(1-\hat{q}),(1-\hat{p}) \hat{q}]$ when $\hat{D}>0$ or $\min [\hat{p} \hat{q}$, $(1-\hat{p})(1-\hat{q})]$ when $\hat{D}<0$. The range of $\hat{D}^{\prime}$ always varies from -1.0 to +1.0 , regardless of the relative allele frequencies at the loci, because it represents the proportion of the maximum value of $\hat{D}$. Maximum values of $\hat{D}^{\prime}=-1.0$ and $\hat{D}^{\prime}=+1.0$ can be obtained when at least one of the coupling and repulsion haplotype frequencies, respectively, take on a zero value; a situation often described as a 'perfect LD' [17].

Estimates of the strength of LD by the coefficient of correlation of allele frequencies ( $r$, also described in the literature as $\Delta$ ) were computed as

$$
\hat{r}=\hat{D} / \sqrt{\hat{p}(1-\hat{p}) \hat{q}(1-\hat{q})} .
$$

Unlike $D^{\prime}$, the limits of the range of $\hat{r}$ fluctuate greatly depending on the allele frequencies. Thus, the maximum possible value of $\hat{r}$ $\left(\hat{r}_{\text {max }}\right)$ is defined as

$$
\hat{r}_{\max }=\hat{D}_{\max } / \sqrt{\hat{p}(1-\hat{p}) \hat{q}(1-\hat{q})}
$$

[15]. Then $\hat{r}$ ranges from -1.0 to +1.0 only if $\hat{p}=\hat{q}=0.5$. Maximum values of $\hat{r}=-1.0$ and $\hat{r}=+1.0$ can be attained only when the product of frequencies of haplotypes in coupling and repulsion, re- spectively, take on a zero value. If $\hat{p}=\hat{q} \neq 0.5$ and $\hat{p} \neq \hat{q}$, then $\hat{r}$ cannot attain values of -1.0 and +1.0 , respectively, given the above chosen criterion to designate coupling haplotype classes. Taking into account the sign of $\hat{r}$ estimates then $\hat{r}^{\prime}=\hat{r} / \hat{r}_{\text {max }}$ is equal to $\hat{D}^{\prime}$ and, therefore, $\hat{r}^{\prime 2}=\hat{D}^{\prime 2}[15,30,31]$.

\section{Simulations}

Sampling distributions of $\hat{D}^{\prime}$ and $\hat{r}$ from two-locus relative haplotype frequencies were obtained by Monte Carlo simulation for different population conditions and varying sample sizes. A total of thirty-six different population conditions were studied: three levels (weak, moderate and strong) of positive and negative $\mathrm{LD}$ and six different combinations of allele frequencies at the two loci ( $p, q$ taking values of $0.5,0.7$ and 0.9$)$. Values of $D^{\prime}$ and $r$ attaining 20,50 and $80 \%$ of the maximum of their respective positive and negative ranges were arbitrarily designated weak $( \pm W)$, moderate $( \pm M)$ and strong $( \pm S)$, respectively, regardless of their biological significance. With respect to $D^{\prime}$, values of LD were invariably $\pm W= \pm 0.2 ; \pm M= \pm 0.5$, and $\pm S= \pm 0.8$, because the limits of its range are frequency independent. In contrast, $r$ values for $\pm W, \pm M$ and $\pm S$ undergo pronounced variations depending on the allele frequencies at the loci (table 1). For each population condition ( $D^{\prime}, p$ and $q$ values), $r$ was computed as

$$
r=D^{\prime} D_{\max } / \sqrt{p(1-p) q(1-q)}
$$

given that $D=D^{\prime} D_{\max }$. For example, if $D^{\prime}=-0.5(=-M)$ and $p=$ $q=0.7$, then $D_{\max }=0.09, r=-0.214$ and $r_{\max }=0.429$. This example shows that values of $D^{\prime}$ and $r$ are equivalent: $D^{\prime}=-0.5(=-M)$ is an intermediate value of the maximum possible value of $D^{\prime}$ with negative sign and $r=-0.214=-1 / 2 r_{\max }(=-M)$. According to the above definitions, two-locus haplotype frequencies for each simulated population were obtained as follows: $p_{11}=D^{\prime} D_{\max }+p q$, $p_{12}=p-p_{11}, p_{21}=q-p_{11}$ and $p_{22}=1-p-q+p_{11}$. Sampling distributions of $\hat{D}^{\prime}$ and $\hat{r}$ were constructed from $n(=1,000)$ random haplotype samples without singletons (i.e. alleles occurring once in the sample) of variable size $N(=50,100,200$ and 400$)$ drawn with replacement from each simulated population and then computing the corresponding 1,000 estimates for each coefficient (see also online suppl. simulation details; for all online suppl. material, see www.karger.com/doi/10.1159/000327732). 
Table 2. Maximum bias of $\hat{D}^{\prime}$ and $\hat{r}$ depending on the relative allele frequencies and population LD

\begin{tabular}{|c|c|c|c|c|c|c|c|c|}
\hline \multirow[t]{2}{*}{ Measure } & \multirow[t]{2}{*}{$\hat{p}$} & \multirow[t]{2}{*}{$\hat{q}$} & \multicolumn{6}{|c|}{ Negative $B_{\max } /$ Positive $B_{\max }$} \\
\hline & & & $-W$ & $-M$ & $-S$ & $+W$ & $+M$ & $+S$ \\
\hline \multirow[t]{6}{*}{$r$} & 0.5 & 0.5 & $-0.800 /+1.200$ & $-0.500 /+1.500$ & $-0.200 /+1.800$ & $-1.200 /+0.800$ & $-1.500 /+0.500$ & $-1.800 /+0.200$ \\
\hline & 0.7 & 0.5 & $-0.524 /+0.786$ & $-0.328 /+0.982$ & $-0.131 /+1.179$ & $-0.786 /+0.524$ & $-0.982 /+0.328$ & $-1.179 /+0.131$ \\
\hline & 0.9 & 0.5 & $-0.266 /+0.400$ & $-0.166 /+0.500$ & $-0.066 /+0.600$ & $-0.400 /+0.266$ & $-0.500 /+0.166$ & $-0.600 /+0.066$ \\
\hline & 0.7 & 0.7 & $-0.343 /+1.086$ & $-0.215 /+1.214$ & $-0.086 /+1.343$ & $-0.629 /+0.800$ & $-0.929 /+0.500$ & $-0.772 /+0.200$ \\
\hline & 0.9 & 0.7 & $-0.174 /+0.553$ & $-0.109 /+0.609$ & $-0.043 /+0.684$ & $-0.320 /+0.407$ & $-0.473 /+0.254$ & $-0.625 /+0.102$ \\
\hline & 0.9 & 0.9 & $-0.089 /+1.022$ & $-0.055 /+1.056$ & $-0.022 /+1.089$ & $-0.311 /+0.800$ & $-0.611 /+0.500$ & $-0.911 /+0.200$ \\
\hline$D^{\prime}$ & all & all & $-0.800 /+1.200$ & $-0.500 /+1.500$ & $-0.200 /+1.800$ & $-1.200 /+0.800$ & $-1.500 /+0.500$ & $-1.800 /+0.200$ \\
\hline
\end{tabular}

\section{Non-Standardised Statistics}

Precision and accuracy of $\hat{D}^{\prime}$ and $\hat{r}$ were first assessed from sampling distributions by means of sampling variances and biases, respectively. The sampling variance $(V)$ and bias $(B)$ of $\hat{D}^{\prime}$ were calculated as follows

$$
V\left(\hat{D}^{\prime}\right)=\frac{1}{n} \sum_{i=1}^{n}\left(\hat{D}_{i}^{\prime}-\overline{\hat{D}^{\prime}}\right)^{2}
$$

and

$$
B\left(\hat{D}^{\prime}\right)=\frac{1}{n} \sum_{i=1}^{n}\left(\hat{D}_{i}^{\prime}-D^{\prime}\right)
$$

Sampling variances, biases and other statistics defined hereafter were similarly computed for $\hat{r}$ by replacing the values of $\hat{D}^{\prime}$ and $D^{\prime}$ used in the definitions with the corresponding $\hat{r}$ and $r$ values, respectively.

The efficiencies of $\hat{D}^{\prime}$ and $\hat{r}$ were estimated by the mean square error (MSE), a measure of the total error of the estimates due to the joint contribution of variances and biases $[32,33]$. The MSE of $\hat{D}^{\prime}$ is given by

$$
\operatorname{MSE}\left(\hat{D}^{\prime}\right)=V\left(\hat{D}^{\prime}\right)+B\left(\hat{D}^{\prime}\right)^{2} .
$$

\section{Standardised Statistics}

Alternative standardised statistics that allow for comparisons of the sampling variation between measures with different ranges were also applied to explore the relative precision and accuracy of $\hat{D}^{\prime}$ and $\hat{r}$. The precision of $\hat{D}^{\prime}$ and $\hat{r}$ was compared using the conventional coefficient of variation. The coefficient of variation $(C V)$ of $\hat{D}^{\prime}$ is

$$
C V\left(\hat{D}^{\prime}\right)=\left[S D\left(\hat{D}^{\prime}\right) / \overline{\hat{D}^{\prime}}\right] \times 100,
$$

where $S D\left(\hat{D}^{\prime}\right)$ is the standard deviation of the distribution of $\hat{D}^{\prime}$.

Assessment of the relative accuracy of $\hat{D}^{\prime}$ and $\hat{r}$ is not a trivial task because the raw biases of $\hat{D}^{\prime}$ and $\hat{r}$ often vary on very different scales. For example, if the population value of the strength of LD between two loci is $+M$ and the relative allele frequencies in a sample taken from the population are $\hat{p}=0.7$ and $\hat{q}=0.5$ then the maximum possible bias $\left(B_{\text {max }}\right)$ of $\hat{D}^{\prime}\left[B_{\text {max }}\left(\hat{D}^{\prime}\right)\right]$ of the positive sign is +0.5 , i.e. the difference between the maximum possible value of positive sign that $\hat{D}^{\prime}$ can take $(+1)$ and its true population value $(+0.5)$. In contrast, the $B_{\max }$ of $\hat{r}\left[B_{\max }(\hat{r})\right]$ of the positive sign is only +0.328 because the maximum possible value of the positive sign that $\hat{r}$ can take and the true population value are +0.655 and +0.327 , respectively (table 1 ). Table 2 shows the $B_{\max }$ of the positive and negative sign that $\hat{D}^{\prime}$ and $\hat{r}$ can attain under simulated population conditions. Comparisons of the accuracy of $\hat{D}^{\prime}$ and $\hat{r}$ were then performed by a new statistic of standardised bias, which is defined as the ratio between the raw bias and the $B_{\max }$. The standardised bias $(S B)$ of $\hat{D}^{\prime}$ is

$$
S B\left(\hat{D}^{\prime}\right)=\frac{1}{n} \sum_{i=1}^{n}\left[\left(\hat{D}_{i}^{\prime}-D^{\prime}\right) / B_{\max }\left(\hat{D}^{\prime}\right)\right],
$$

where $B_{\max }\left(\hat{D}^{\prime}\right)$ is $\left(-1-D^{\prime}\right)$ if $\left(\hat{D}^{\prime}{ }_{i}-D^{\prime}\right)<0$ and $\left(1-D^{\prime}\right)$ if $\left(\hat{D}^{\prime}{ }_{i}-\right.$ $\left.D^{\prime}\right)>0$. Note that $B_{\text {max }}(\hat{r})$ is $\left(-\hat{r}_{\text {max }}-r\right)$ if $\left(\hat{r}_{i}-r\right)<0$ and $\left(\hat{r}_{\text {max }}-r\right)$ if $\left(\hat{r}_{i}-r\right)>0$. Estimates of $S B\left(\hat{D}^{\prime}\right)$ and $S B(\hat{r})$ expressed in standard deviation units were also obtained. The $S B\left(\hat{D}^{\prime}\right)$ in standard deviation units $(s d u)$ is given by

$$
S B\left(D^{\prime}\right)_{s d u}=S B\left(\hat{D}^{\prime}\right) / \sqrt{V\left(\hat{D}^{\prime}\right)} .
$$

The relative efficiency of each measure of LD was estimated by means of the relative error, which was computed as the ratio (expressed in percentage) between the root of the MSE and the true value of disequilibrium in absolute value. The relative error ( RelError) of $\hat{D}^{\prime}$ is given by

$$
\operatorname{RelError}\left(\hat{D}^{\prime}\right)=\left(\sqrt{\operatorname{MSE}\left(\hat{D}^{\prime}\right)} /\left|D^{\prime}\right|\right) \times 100 \text {. }
$$

\section{Sampling Distributions of Non-Standardised and}

Standardised Statistics

Previous comparative studies on the precision and accuracy of $\hat{D}^{\prime}$ and $\hat{r}$ were based on the analysis of point estimates of their sampling variances and biases [21-23]. However, variances and biases of the estimates of $D^{\prime}$ and $r$ are random variables and, therefore, point estimates might not be representative of their sampling distributions. In this work, empirical sampling distributions of non-standardised and standardised statistics used for the measurement of precision, accuracy and efficiency of each coefficient were eventually obtained from 50 replicate sampling distributions (1,000 haplotype samples each) of $\hat{D}^{\prime}$ and $\hat{r}$ for each population condition and sample size. 


\section{Results}

Simulations provided sufficient information for a detailed evaluation and comparison of the relative performance of $\hat{D}^{\prime}$ and $\hat{r}$ under each of the 144 combinations of levels of disequilibrium ( $\pm W, \pm M$ and $\pm S$ ), allele frequencies at the two loci ( $p, q$ ranging from 0.5 to 0.9 ) and sample sizes $(N=50,100,200$ and 400$)$. For the purpose of brevity, data analyses were based on mean values of the sampling distribution of the statistics used to assess the precision, accuracy and efficiency of $\hat{D}^{\prime}$ and $\hat{r}$ under each population condition and sample size. Mean values of sampling variances, raw biases, coefficients of variation, standardised biases, MSEs and relative errors of $\hat{D}^{\prime}$ and $\hat{r}$ across the scenarios studied are available as supplementary material (online suppl. tables 1-6).

\section{Precision and Accuracy of $\hat{D}^{\prime}$ and $\hat{r}$ by}

\section{Non-Standardised Statistics}

As expected, the variances of both coefficients showed an overall increase for smaller sample sizes, lower allele frequencies and weak LD, but they were systematically higher for $\hat{D}^{\prime}$ than for $\hat{r}$ under all population conditions and sample sizes (sign test, $\mathrm{p}<0.001$; table 3 and online suppl. table 1). With respect to the bias, $\hat{D}^{\prime}$ was nearly always biased downward (143 out of 144 cases) when the true LD was negative and biased upward (137 out of 144 cases) when the true LD was positive (online suppl. table 2 ). In contrast, $\hat{r}$ was generally biased upward (130 out of 144 cases) for negative LD and biased downward (122 out of 144 cases) for positive LD (online suppl. table 2). These trends in bias of $\hat{D}^{\prime}$ and $\hat{r}$ were found to be highly significant (sign test, $\mathrm{p}<0.001$ ). It follows, therefore, that $\hat{D}^{\prime}$ tends to overestimate the strength of LD, whereas $\hat{r}$ tends to underestimate it. The bias (expressed in absolute value) was generally higher for $\hat{D}^{\prime}$ (124 out of 144 cases) compared with $\hat{r}$ (sign test, $\mathrm{p}<0.001$; online suppl. table 2). Descriptive measurements (range, median and mean) of the distribution of ratios between variances and between biases (in absolute value) of both coefficients give us a quantitative view of the extent of these discrepancies (table 3). Both types of ratios were often far greater than 1.0. In many cases, biases of $\hat{D}^{\prime}$ were even several orders of magnitude greater than those of $\hat{r}$, and ratios between biases of $\hat{D}^{\prime}$ and $\hat{r}$ across the scenarios studied averaged ( \pm SE, standard error) $139 \pm 37$. By way of illustration, figure 1 provides a visual perspective of the magnitude of the differences in ratios of variances and biases of $\hat{D}^{\prime}$ and $\hat{r}$ (in logarithmic units) across the complete set of population conditions analysed when $N=100$.

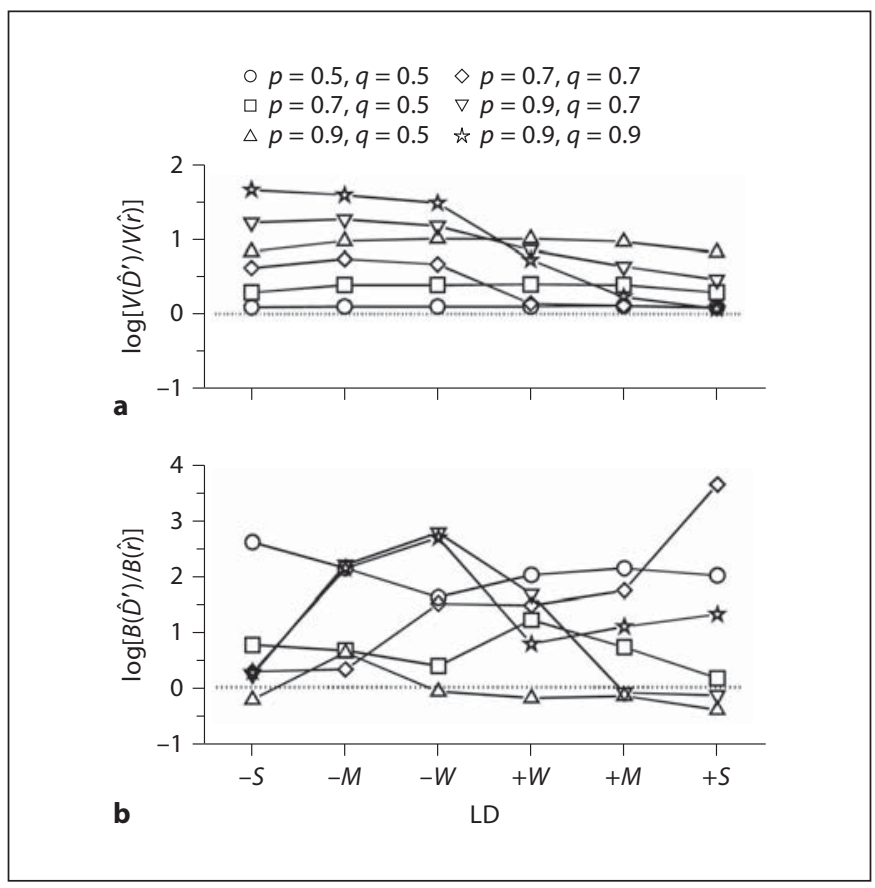

Fig. 1. Relative precision and accuracy of $\hat{D}^{\prime}$ and $\hat{r}$ under different simulated population conditions and sample sizes of 100 haplotypes by non-standardised statistics. a Logarithm of the ratios of variances $(V)$ of $\hat{D}^{\prime}$ and $\hat{r}$. $\mathbf{b}$ Logarithm of the ratios of biases $(B)$ of $\hat{D}^{\prime}$ and $\hat{r}$ (in absolute value).

Table 3. Precision and accuracy of $\hat{D}^{\prime}$ and $\hat{r}$ across simulated scenarios by non-standardised statistics

\begin{tabular}{llllc}
\hline Statistic & \multicolumn{2}{l}{ Range } & Median & Mean \pm SE \\
\cline { 2 - 3 } & minimum & maximum & & \\
\hline$V\left(\hat{D}^{\prime}\right)$ & 0.001 & 0.334 & 0.022 & $0.051 \pm 0.006$ \\
$V(\hat{r})$ & 0.001 & 0.045 & 0.005 & $0.008 \pm 0.001$ \\
$\left|B\left(\hat{D}^{\prime}\right)\right|$ & 0.000 & 0.397 & 0.007 & $0.026 \pm 0.004$ \\
$|B(\hat{r})|$ & 0.000 & 0.005 & 0.000 & $0.001 \pm 0.000$ \\
$V\left(\hat{D}^{\prime}\right) / V(\hat{r})$ & 1.13 & 69.86 & 2.89 & $8.12 \pm 1.04$ \\
$\left|B\left(\hat{D}^{\prime}\right)\right| /|B(\hat{r})|$ & 0.06 & 4,560 & 18.18 & $139 \pm 37$ \\
\hline
\end{tabular}

$V=$ Sampling variance; $B=$ sampling bias.

\section{Precision and Accuracy of $\hat{D}^{\prime}$ and $\hat{r}$ by Standardised}

\section{Statistics}

Differences between coefficients of variation of $\hat{D}^{\prime}$ and $\hat{r}$ did not follow any consistent trend showing either a disadvantage or an advantage of $\hat{D}^{\prime}$ with respect to $\hat{r}$ (sign test, $\mathrm{p}=0.63$; online suppl. table 3 ). Simulations also 


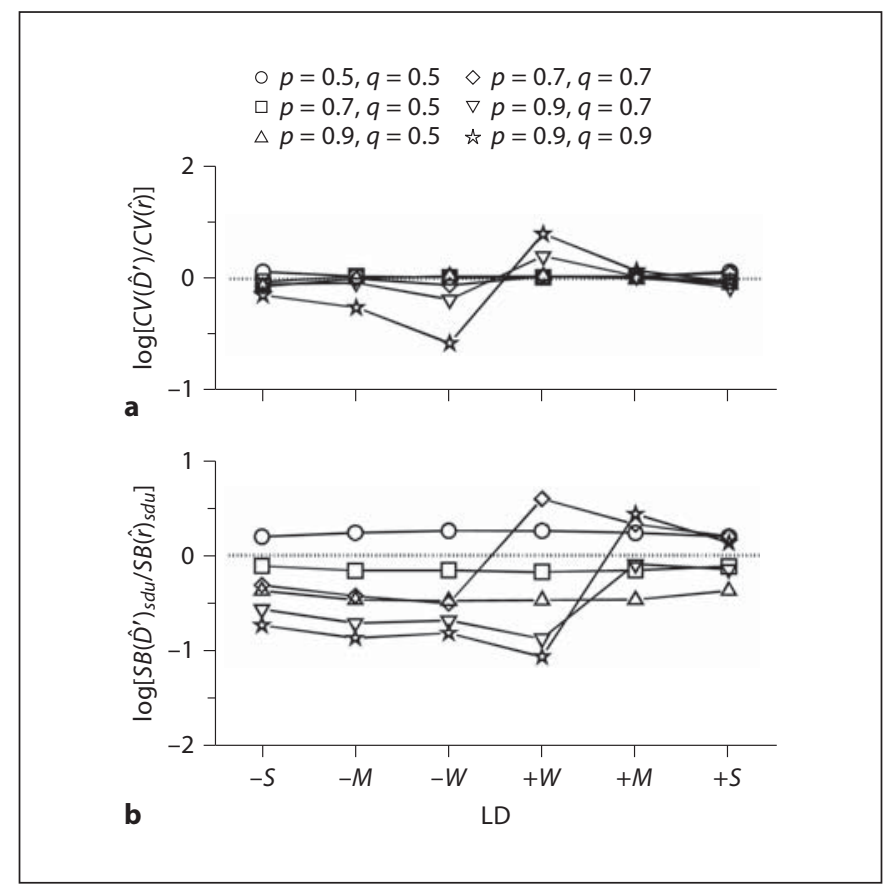

Fig. 2. Relative precision and accuracy of $\hat{D}^{\prime}$ and $\hat{r}$ over different simulated population conditions and sample sizes of 100 haplotypes by standardised statistics. a Logarithm of the ratios of coefficients of variation $(C V)$ of $\hat{D}^{\prime}$ and $\hat{r}$. $\mathbf{b}$ Logarithm of the ratios of standardised biases $(S B)$ of $\hat{D}^{\prime}$ and $\hat{r}$ (expressed in standard deviation units, $s d u)$.

showed that discrepancies detected between the coefficients of variation of $\hat{D}^{\prime}$ and $\hat{r}$ were in general insignificant (fig. 2a). In fact, median and mean values of the distribution of ratios between coefficients of variation were 1.0 (table 4). Accordingly, the Friedman two-way analysis of variance by ranks showed that coefficients of variation of $\hat{D}^{\prime}$ and $\hat{r}$ were not significantly different from each other through the cases studied ( $\mathrm{p}=0.56)$. Secondly, standardised biases were generally higher (124 out of 144 cases) for $\hat{D}^{\prime}$ than for $\hat{r}$ (sign test, $\mathrm{p}<0.001$; online suppl. table 4), but the differences between coefficients decreased dramatically when compared with raw biases. On average, the ratio between standardised biases (in absolute value) of $\hat{D}^{\prime}$ and $\hat{r}$ was $1.44 \pm 0.08$ (table 4 ), a value that is approximately 100 -fold lower than the average ratio of non-standardised biases. It should also be considered that previous simulation data have shown that differential bias among estimates of $D^{\prime}$ can sometimes be explained by their unequal variances [21]. Accordingly, a possible relationship between standardised biases and variances for both $\hat{D}^{\prime}$ and $\hat{r}$ was tested in our simulations by calculation of Spearman's nonparametric coefficient
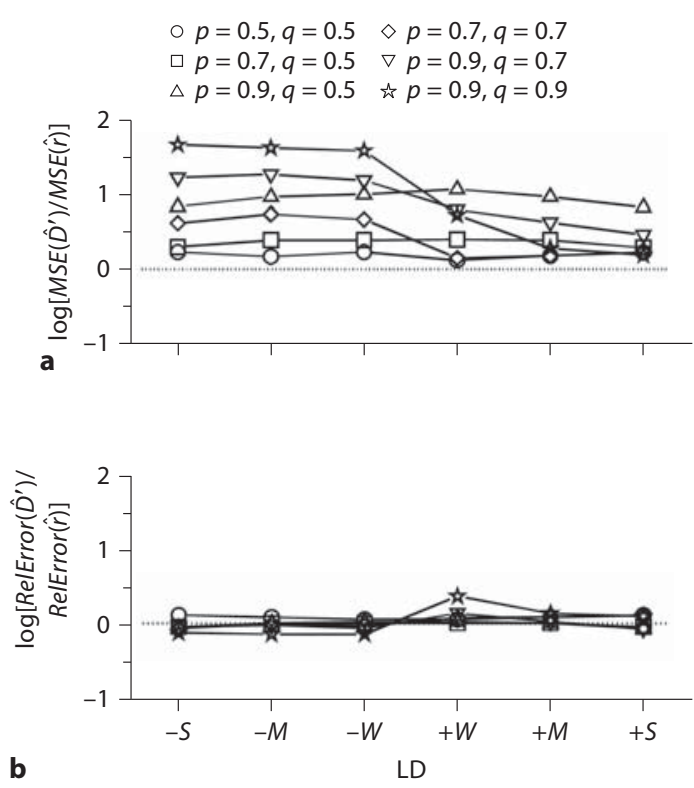

Fig. 3. Relative magnitude of the efficiency of $\hat{D}^{\prime}$ and $\hat{r}$ over different simulated population conditions and sample sizes of 100 haplotypes. a Logarithm of the ratios of mean square errors (MSE) of $\hat{D}^{\prime}$ and $\hat{r}$. b Logarithm of the ratios between relative errors (RelError) of $\hat{D}^{\prime}$ and $\hat{r}$.

of rank correlation $\left(r_{s}\right)$. We found a statistically significant positive correlation only between values of standardised bias and variances of $\hat{D}^{\prime}\left(r_{s}=+0.25, \mathrm{p}<0.01\right.$, $n=144$ for $\hat{D}^{\prime} ; r_{s}=+0.08, \mathrm{p}=0.37, n=144$ for $\left.\hat{r}\right)$. Estimated biases can then be expressed in $s d u$ to minimise the influence of the variance [33]. By doing so, it was found that the standardised bias (in $s d u$ ) of $\hat{D}^{\prime}$ was much smaller than that of $\hat{r}$ over a large portion (100 out of 144) of cases (sign test, $p<0.001$; table 4 ; fig. $2 b$ ), and the median distribution value for their ratios was 0.68 (Friedman test, $\mathrm{p}<0.001)$.

\section{Efficiency of $\hat{D}^{\prime}$ and $\hat{r}$}

MSEs were always higher for $\hat{D}^{\prime}$ than for $\hat{r}$ across simulated scenarios (sign test, $\mathrm{p}<0.001$; online suppl. table 5) and, as a result, their ratios were always higher than 1.0 (table 5; fig. 3a). However, the sampling variance was systematically the dominant component of the total error. As shown in table 5, the median and mean of the magnitude of the error explained by the variance were greater than $94 \%$ for both measures. Accordingly, the behaviour of the MSE and the variance was very similar across the 
Table 4. Relative precision and accuracy of $\hat{D}^{\prime}$ and $\hat{r}$ across simulated scenarios by standardised statistics

\begin{tabular}{lllll}
\hline Ratio & \multicolumn{2}{l}{ Range } & \multirow{2}{*}{ Median Mean \pm SE } \\
\cline { 2 - 4 } & minimum maximum & & \\
\hline$C V\left(\hat{D}^{\prime}\right) / C V(\hat{r})$ & 0.16 & 9.20 & 1.00 & $1.03 \pm 0.06$ \\
$\left|S B\left(\hat{D}^{\prime}\right)\right| /|S B(\hat{r})|$ & 0.20 & 6.44 & 1.09 & $1.44 \pm 0.08$ \\
$\left|S B\left(\hat{D}^{\prime}\right)_{s d u}\right| /\left|S B(\hat{r})_{s d u}\right|$ & 0.07 & 5.03 & 0.68 & $0.96 \pm 0.08$ \\
\hline
\end{tabular}

$C V=$ Coefficient of variation; $S B=$ standardised bias; $s d u=$ standard deviation unit.

Table 5. Efficiency of $\hat{D}^{\prime}$ and $\hat{r}$ across simulated scenarios

\begin{tabular}{lcccc}
\hline Statistic & \multicolumn{2}{l}{ Range } & Median & Mean \pm SE \\
\cline { 2 - 4 } & $\begin{array}{l}\text { mini- } \\
\text { mum }\end{array}$ & $\begin{array}{l}\text { maxi- } \\
\text { mum }\end{array}$ & & \\
\hline$M S E\left(\hat{D}^{\prime}\right)$ & 0.002 & 0.473 & 0.022 & $0.054 \pm 0.007$ \\
$M S E(\hat{r})$ & 0.001 & 0.045 & 0.005 & $0.008 \pm 0.001$ \\
$M S E\left(\hat{D}^{\prime}\right) / M S E(\hat{r})$ & 1.17 & 69.83 & 2.95 & $8.46 \pm 1.08$ \\
$V\left(\hat{D}^{\prime}\right) / M S E\left(\hat{D}^{\prime}\right)^{\mathrm{a}}$ & 66.74 & 100 & 99.91 & $94.12 \pm 0.79$ \\
$\operatorname{V}(\hat{r}) / M S E(\hat{r})^{\mathrm{a}}$ & 99.87 & 100 & 99.99 & $99.98 \pm 0.02$ \\
$\operatorname{RelError}\left(\hat{D}^{\prime}\right)$ & 4.87 & 344.0 & 35.05 & $57.90 \pm 5.39$ \\
$\operatorname{RelError}(\hat{r})$ & 3.75 & 592.7 & 34.82 & $58.50 \pm 6.45$ \\
$\operatorname{RelError}\left(\hat{D}^{\prime}\right) /$ & 0.53 & 2.90 & 1.02 & $1.07 \pm 0.02$ \\
$\quad \operatorname{RelError}(\hat{r})$ & & & & \\
\hline
\end{tabular}

$M S E=$ Mean square error; $V=$ sampling variance; RelError $=$ relative error.

a Values are given as percentages.

sample sizes and population conditions studied (cf. fig. 1a and 3a). On the other hand, an analysis of the distribution of the relative error of $\hat{D}^{\prime}$ and $\hat{r}$ revealed several relevant aspects (table 5; online suppl. table 6). First, the relative error of the estimates of LD was generally very considerable, with mean values over cases of $58 \%$ for both $\hat{D}^{\prime}$ and $\hat{r}$, but fluctuated markedly across the population conditions and sample sizes studied. Expectedly, the relative error often decreased as the sample size, the level of polymorphism at the loci and the strength of LD increased. To illustrate this, variations in relative error of $\hat{D}^{\prime}$ for extreme sample sizes were as follows: for a smaller sample size $(N=50)$, errors ranged from 14 to $344 \%$ and averaged $93 \pm 15 \%$, whereas for a larger sample $(N=400)$, they ranged from 5 to $156 \%$ and averaged $31 \pm 5.2 \%$. However, relative errors and their fluctuations decreased con- siderably in more favorable situations. For example, if $N=400$ and the strength of $\mathrm{LD}$ is $+S$, errors then ranged from 5 to $12 \%$ and averaged $8 \pm 1 \%$. Secondly, differences in relative error between $\hat{D}^{\prime}$ and $\hat{r}$ did not follow a significant trend (sign test, $\mathrm{p}=0.08$; relative errors were higher for $\hat{D}^{\prime}$ that for $\hat{r}$ in 83 out of 144 cases). It is noteworthy that although the probability of the sign test was relatively close to the level of significance $(\alpha=0.05)$, differences between coefficients were, in general, quantitatively negligible to the point that the median of ratio distribution between relative errors of $\hat{D}^{\prime}$ and $\hat{r}$ achieved a value of only 1.02 (table 5; fig. 3b). In addition, the differences in efficiency between measures were not statistically significant for any of the cases studied (Friedman test, $\mathrm{p}=0.07$ ).

\section{Sign-Dependent Sampling Distributions of $\hat{D}^{\prime}$ and $\hat{r}$}

The variance of $\hat{D}^{\prime}$ was generally higher for negative than positive LD (sign test, $\mathrm{p}<0.01$ ) while for $\hat{r}$ the opposite was true (sign test, $\mathrm{p}<0.001$; online suppl. table 1 ). However, a more detailed analysis of the observations showed that significant sign-dependent trends were caused rather by the proportion of cases (36/72) where allele frequencies at both loci depart from 0.5 (sign test, $\mathrm{p}<0.001$ for both coefficients) than by the remaining ones (sign test, $\mathrm{p}=0.27$ for $\hat{D}^{\prime}$ and $\mathrm{p}=0.30$ for $\hat{r}$ ). Signdependent differences were quantitatively relevant because the variances of negative $\hat{D}^{\prime}$ were, on average, approximately fivefold higher than the variances of positive $\hat{D}^{\prime}$, when allele frequencies at both loci differed from 0.5 (Mann-Whitney test, $\mathrm{p}<0.001$ ). Importantly, sign-dependent trends for both coefficients become consistent by applying coefficients of variation. Thus, coefficients of variation of $\hat{D}^{\prime}$ and $\hat{r}$ were generally higher for negative than for positive LD (32 and 36 of 36 cases, respectively), when allele frequencies at both loci were not 0.5 (sign test, $\mathrm{p}<0.001$; online suppl. table 3).

\section{Discussion}

The present study provides new insights to assess the optimal use and application of the two most commonly used measures of LD in line with the relative strengths and weaknesses of their statistical properties. With the application of standardised statistics, we found that $\hat{r}$ does not tend to be significantly more precise, accurate or efficient than $D^{\prime}$ across population conditions and sample sizes. These observations suggest that both coefficients capture similar information from haplotype data, 
but at a very different scale or range. This is not a surprising result when one takes into account that $D^{\prime}$ is equal to $r$ standardised by its maximum possible value $\left(r_{\max }\right)$ given the sign of deviations from random association and allele frequencies at the two loci $[15,30,34]$. The close relationship between both coefficients becomes empirically clearer when the range of $r$ (or $r^{2}$ ) across locus pairs is comparable to $D^{\prime}$. For example, it has been reported that the average decay of LD between SNP pairs with physical distance in gene regions of the human genome as measured by $D^{\prime}$ and $r^{2}$ becomes fairly similar using SNPs matched for allele frequency [35]. As can be deduced from table $1, r^{2}$ has the same range as $D^{\prime}$ when $r>0$ and $p=q$. It follows that most of the inconsistencies often observed between estimates of $D^{\prime}$ and $r$ across genomes is likely a byproduct of direct comparisons between measures with different ranges.

Our observations show that the importance of bias in the estimation of the strength of LD has clearly been overemphasised because the contribution of the bias to the total error of $\hat{D}^{\prime}$ and $\hat{r}$ was considerably smaller $(<6 \%$ on average) than that of the variance across all of the population conditions and sample sizes analysed. Hence, efforts to reduce the finite-sample bias using the existing bias-correction techniques [21,36-38] will likely have a reduced benefit for the level of efficiency of $\hat{D}^{\prime}$ and $\hat{r}$. Furthermore, bias-correction techniques might have negative consequences on the principal source of error because bias improvements are often outweighed to varying degrees by decreased precision due to the usual tradeoff between bias and variance [33]. More effective improvements in the efficiency of the estimates of LD could be achieved in practice trying to reduce the large sampling variances by the choice of loci with a higher level of polymorphism and/or the use of large experimental sample sizes. On the other hand, increased precision is one of the advantages of estimates of LD with a positive sign. Specifically, we have found that variances were, on average, approximately fivefold lower for $\hat{D}^{\prime}$ of positive signs than for $\hat{D}^{\prime}$ of negative signs when allele frequencies at both loci are not intermediate, which is likely due to the signdependent asymmetry of $\hat{D}_{\max }$ [39]. This finding is in qualitative agreement with previously reported observations based on the asymptotic variance of $\hat{D}^{\prime}$ [25].

The present observations substantially change the current view on the relative merits of $D^{\prime}$ and $r$ for estimating and comparing the levels of $L D$ across genomes. It can no longer be justified to maintain that $D^{\prime}$ tends to overestimate the importance of LD in populations and leads to erroneous comparisons of $\mathrm{LD}$ among locus pairs be- cause it provides estimates that are strongly biased for small samples. On the contrary, $D^{\prime}$ has an advantage over $r$ in the fact that its range of variation is always the same (from -1.0 to +1.0 ) regardless of the allele frequencies at loci. It allow us to assess more exactly the strength of $\mathrm{LD}$ between a given locus pair and to establish differences in the strength of LD across pairs of loci within and among populations $[8,20,40]$. In contrast, the limits of the range of $r$ are frequency dependent, which has two main negative consequences. Firstly, the measure $r$ can give the false impression that the strength of LD between pairs of loci is lower than it really is, and thus to underestimate the importance of the phenomenon in populations. For example, the value of $r$ for pairs of loci in strong negative $\mathrm{LD}(-S)$ is only -0.089 when $p=q=0.9$, whereas the corresponding value of $D^{\prime}$ is -0.800 (table 1 ). Secondly, values of $r$ do not proportionately increase with the increasing strength of LD regardless of the allele frequencies, which affects the validity of comparisons of the extent of LD across pairs of loci. Paradoxically, the value of $r$ can decrease as the strength of LD actually increases depending on the allele frequencies at the loci. Table 1 provides an illustration of this anomalous behaviour such as, for example, that values of $r$ of -0.200 and -0.089 correspond to weak $(-W)$ and strong $(-S)$ LD when $p=q=0.5$ and $p=q=0.9$, respectively.

The advantage of $D^{\prime}$ over $r$ for interpreting some patterns of $\mathrm{LD}$ along genomes is widely recognised. By way of illustration, $D^{\prime}$ seems to be more directly related to the recombination fraction among pairs of loci than coefficients such as $r$ that have a frequency-dependent range $[4$, $36,40,41]$. An example on the advantages of $D^{\prime}$ over $r$ for detecting patterns of the strength of LD between SNPs with physical distance from the HapMap Project data [2] is provided as an online supplementary example. In addition, $D^{\prime}$ can be useful to identify genome regions without or with minimal historical recombination $[1,12,17$, 35]. If perfect ancestral LD between two genetic markers has not been disrupted during the history of the population then at most three out of the four possible two-locus haplotypes are observed in the sample. Consequently, maximum values of $D^{\prime}= \pm 1$ provide a useful indication of the absence of any historical recombination, and deviations from $D^{\prime}= \pm 1$ thus give evidence for at least one historical recombination event. However, a major criticism against the use of $D^{\prime}$ is precisely that it tends to inflate the real levels of $L D$ in populations because the high frequency of $D^{\prime}= \pm 1$ commonly found in experimental studies could be merely due to the absence by sampling error of haplotypes with low expectations $[9,12,17,19,29$, 
42]. Note, however, that $r$ also achieves its maximum possible value $\left(r_{\max }\right)$ if one or two of the four haplotypes are not observed. It seems, therefore, that $\hat{D}^{\prime}$ and $r$ suffer from the same problem but on a different scale.

It is often assumed that $r$ (or $r^{2}$ ) has several statistical properties that make it a more desirable measure of $\mathrm{LD}$ in two main contexts. Firstly, $r$ has a very close relationship with the $\chi^{2}$ test which is particularly useful for genetic mapping of disease susceptibility genes by association because in order to achieve the same power to detect association at the marker locus as we would have at the locus causal, we need to increase our sample size by a factor of $1 / r^{2}[9,14,17,19,23,43]$. But this does not mean that $r$ is a better measure of the strength of $\mathrm{LD}$ than $D^{\prime}$, even in the context of gene mapping $[4,5,44,45]$. It should be emphasised that $D^{\prime}$ with a positive sign is equal to the allelic association metric measure $(\rho)$ [46-48], which was first introduced as a powerful tool to identify a region for positional cloning of a disease gene and it has been considered the 'optimal measure of allelic association' for describing patterns of LD both empirically and from the population genetics theory $[5,44,45]$. Secondly, $r$ has been traditionally used to study the effect of finite population size and recombination on $\operatorname{LD}[7,9,34,49]$. Hill and Robertson [7] carried out extensive simulation experiments and showed that the average values of $\hat{r}^{2}$ under a model of selectively neutral evolution are almost entirely determined by $N_{e} c$ at equilibrium, where $N_{e}$ is the effective population size and $c$ is the recombination rate between two loci. This relevant conclusion becomes consistent with the principle that there is no LD measure completely independent of the allele frequencies [20], taking into account that it does not refer to individual estimates and the range of $r^{2}$ is much smaller than that of $r$. More recently, it was reported that the evolutionary variances of estimates of $r^{2}$ from simulated populations under neutral evolution were often considerably smaller as compared to $D^{\prime}$ estimates [9]. However, this difference does not hold when the evolutionary variations of both coefficients are more correctly compared by using coefficients of variation [8]. It seems, therefore, that $r^{2}$ has the advantage that its expected value by genetic drift approaches $N_{e} c$, but it also has all the above-mentioned disadvantages of a measure with a frequency-dependent range.

\section{Acknowledgements}

We are grateful to two anonymous reviewers for providing insightful comments and suggestions. This research was partly supported by grants PGIDIT03PXIC20003PN and PGIDIT04RMA261014PR (Xunta de Galicia, Spain).

\section{References}

1 Slatkin M: Linkage disequilibrium - understanding the evolutionary past and mapping the medical future. Nat Rev Genet 2008;9: 477-485.

-2 The International HapMap Consortium: A second generation human haplotype map of over 3.1 million SNPs. Nature 2007;449:851862.

3 Hedrick PW, Jain S, Holden L: Multilocus systems in evolution. Evol Biol 1978;11:101182.

4 Devlin B, Risch N: A comparison of linkage disequilibrium measures for fine-scale mapping. Genomics 1995;29:311-322.

5 Morton NE, Zhang W, Taillon-Miller P, Ennis S, Kwok PY, Collins A: The optimal measure of allelic association. Proc Natl Acad Sci USA 2001;98:5217-5221.

6 Lewontin RC: The interaction of selection and linkage. I. General considerations: heterotic models. Genetics 1964;49:49-67.

7 Hill WG, Robertson A: Linkage disequilibrium in finite populations. Theor Appl Genet 1968;38:226-231.
8 Hedrick PW: Gametic disequilibrium measures: proceed with caution. Genetics 1987; 117:331-341.

9 Pritchard JK, Przeworski M: Linkage disequilibrium in humans: models and data. Am J Hum Genet 2001;69:1-14.

10 Jorde LB, Bamshad M: Questioning evidence for recombination in human mitochondrial DNA. Science 2000;288:1931a.

11 Kumar S, Hedrick PW, Dowling T, Stoneking M: Questioning evidence for recombination in human mitochondrial DNA. Science 2000;288:1931a.

-12 Gabriel SB, Schaffner SF, Nguyen H, Moore J M, Roy J, Blumenstiel B, Higgins J, DeFelice M, Lochner A, Faggart M, Liu-Cordero SN, Rotimi C, Adeyemo A, Cooper R, Ward R, Lander ES, Daly MJ: The structure of haplotype blocks in the human genome. Science 2002;296:2225-2229.

13 Sabatti C, Risch N: Homozygosity and linkage disequilibrium. Genetics 2002;160:17071719.

14 Weiss KM, Clark AG: Linkage disequilibrium and the mapping of complex human traits. Trends Genet 2002;18:19-24.
15 Zapata C, Alvarez G: Testing for homogeneity of gametic disequilibrium among populations. Evolution 1997;51:606-607.

16 Jorde LB: Linkage disequilibrium and the search for complex disease genes. Genome Res 2000;10:1435-1444.

17 Ardlie K, Kruglyak L, Seielstad M: Patterns of linkage disequilibrium in the human genome. Nat Rev Genet 2002;3:299-309.

18 Flint-Garcia SA, Thornsberry JM, Buckler IV ES: Structure of linkage disequilibrium in plants. Annu Rev Plant Biol 2002;54:357374.

19 Mueller JC: Linkage disequilibrium for different scales and applications. Brief Bioinform 2004;5:355-364.

20 Lewontin RC: On measures of gametic disequilibrium. Genetics 1988;120:849-852.

-21 Teare MD, Dunning AM, Durocher F, Rennart G, Easton DF: Sampling distribution of summary linkage disequilibrium measures. Ann Hum Genet 2002;66:223-233.

-22 Terwilliger JD, Haghighi F, Hiekkalinna TS, Göring HHH: A bias-ed assessment of the use of SNPs in human complex traits. Curr Opin Genet Dev 2002;12:726-734. 
-23 Terwilliger JD, Hiekkalinna T: An utter refutation of the 'Fundamental Theorem of the HapMap'. Eur J Hum Genet 2006;14:426437.

24 Tishkoff SA, Verrelli BC: Role of evolutionary history on haplotype block structure in the human genome: implications for disease mapping. Curr Opin Genet Dev 2003;13: 569-575.

25 Zapata C, Alvarez G, Carollo C: Approximate variance of the standardized measure of gametic disequilibrium $D^{\prime}$. Am J Hum Genet 1997;61:771-774.

-26 Langley CH, Tobari YN, Kojima K: Linkage disequilibrium in natural populations of Drosophila melanogaster. Genetics 1974;78: 921-936.

-27 Brown AHD: Sample sizes required to detect linkage disequilibrium between two or three loci. Theor Pop Biol 1975;8:184-201.

28 Thompson EA, Deeb S, Walker D, Motulsky AG: The detection of linkage disequilibrium between closely linked markers: RFLPs at the AI-CIII apolipoprotein genes. Am J Hum Genet 1988;42:113-124.

-29 Zapata C, Alvarez G: On the detection of non-random associations between DNA polymorphisms in natural populations of Drosophila. Mol Biol Evol 1993;10:823-841.

30 Hedrick PW, Kumar S: Mutation and linkage disequilibrium in human mtDNA. Eur J Hum Genet 2001;9:969-972.

- 31 VanLiere JM, Rosenberg NA: Mathematical properties of the $r^{2}$ measure of linkage disequilibrium. Theor Pop Biol 2008;74:130137.
32 Elandt-Johnson RC: Probability Models and Statistical Methods in Genetics. New York, John Wiley \& Sons, 1971.

33 Efron B, Tibshirani RJ: An Introduction to the Bootstrap. New York, Chapman \& Hall, 1993.

34 Hedrick PW: Genetics of Populations. Boston, Jones and Bartlett Publishers, 2005.

35 Eberle MA, Rieder MJ, Kruglyak L, Nickerson DA: Allele frequency matching between SNPs reveals an excess of linkage disequilibrium in genic regions of the human genome. PLoS Genet 2006;2:1319-1327.

36 Guo S-W: Linkage disequilibrium measures for fine-scale mapping: a comparison. Hum Hered 1997;47:301-314.

37 Varilo T, Paunio T, Parker A, Perola M, Meyer J, Terwilliger JD, Peltonen L: The interval of linkage disequilibrium (LD) detected with microsatellite and SNP markers in chromosomes of Finnish populations with different histories. Hum Mol Biol 2003;12:51-59.

38 Tsai H-J, Sun G, Smelser D, Viali S, Tufa J, Jin L, Weeks DE, McGarvey S, Deka R: Distribution of genome-wide linkage disequilibrium based on microsatellite loci in the Samoan population. Hum Genom 2004;1:327-334.

39 Chakraborty R: Detection of nonrandom association of alleles from the distribution of the number of heterozygous loci in a sample. Genetics 1984;108:719-731

40 Hedrick PW: Inference of recombinational hotspots using gametic disequilibrium values. Heredity 1988;60:435-438.

41 Devlin B, Roeder K, Otto C, Tiobech S, Byerkey W: Genome-wide distribution and linkage disequilibrium in the population of Palau and its implications for gene flow in Remote Oceania. Hum Genet 2001; 108:521528.
42 Hedrick PW, Thomson G: A two-locus neutrality test: applications to humans, E. coli and lodgepole pine. Genetics 1986;112:135156

43 Carlson CS, Eberle MA., Rieder MJ, Smith JD, Kruglyak L, Nickerson D: Additional SNPs and linkage-disequilibrium analyses are necessary for whole-genome association studies in humans. Nat Genet 2003;33:518521.

44 Collins A, Morton NE: Mapping a disease locus by allelic association. Proc Natl Acad Sci USA 1998;95:1741-1745.

45 Maniatis N, Morton NE, Gibson J, Xu C-F, Hosking LK, Collins A: The optimal measure of linkage disequilibrium reduces error in association mapping of affection status. Hum Mol Genet 2005; 14:45-153.

46 Shete S: A note on the optimal measure of allelic association. Ann Hum Genet 2003;67: 189-191.

47 Tapper WJ, Maniatis N, Morton NE, Collins A: A metric linkage disequilibrium map of a human chromosome. Ann Hum Genet 2003; 67:487-494.

48 Mangin B, Garnier-Géré P, Cierco-Ayrolles C: The estimator of the optimal measure of allelic association: mean, variance and probability distribution when the sample size tends to infinite. Stat Appl Genet Mol Biol $2008 ; 7: 1-23$

49 Hudson RR: Linkage disequilibrium and recombination; in Bishop BD, Cannings C (eds): Handbook of Statistical Genetics. New York, Willey \& Sons, 2001, pp 309-324. 